\title{
A case of mushroom-shaped anaplastic oligodendroglioma resembling meningioma and arteriovenous malformation: Inadequacies of diagnostic imaging
}

\author{
YAOLING LIU $^{1,2}$, KANG YANG $^{1}, \mathrm{XU} \mathrm{SUN}^{1}, \mathrm{XINYU} \mathrm{LI}^{1}, \mathrm{MINGHAI} \mathrm{WEI}^{1}$, \\ XIANG'EN SHI $^{2}$, NINGWEI CHE ${ }^{1}$ and JIAN YIN ${ }^{1}$ \\ ${ }^{1}$ Department of Neurosurgery, The Second Affiliated Hospital of Dalian Medical University, \\ Dalian, Liaoning 116044; ${ }^{2}$ Department of Neurosurgery, Affiliated Fuxing Hospital, \\ The Capital University of Medical Sciences, Beijing 100038, P.R. China
}

Received December 29, 2014; Accepted June 29, 2015

DOI: $10.3892 / \mathrm{etm} .2015 .2676$

\begin{abstract}
Magnetic resonance imaging (MRI) is the most widely discussed and clinically employed method for the differential diagnosis of oligodendrogliomas; however, MRI occasionally produces unclear results that can hinder a definitive oligodendroglioma diagnosis. The present study describes the case of a 34-year-old man that suffered from headache and right upper-extremity weakness for 2 months. Based on the presurgical evaluation, it was suggested that the patient had a World Health Organization (WHO) grade II-II glioma, meningioma or arteriovenous malformation (AVM), with unclear radiological manifestations. Postsurgical pathological assessment confirmed the tumor to be an anaplastic oligodendroglioma (WHO grade III). This case is notable due to the confusing radiological manifestation of a mushroom-shaped anaplastic oligodendroglioma in the parietal-temporal-occipital region, which provided a potential source of misdiagnosis for meningioma and AVM.
\end{abstract}

\section{Introduction}

Oligodendroglioma is the third most common type of intracranial glioma and originates from neuroepithelial cells, accounting for $2-5 \%$ of primary brain tumors and $4-15 \%$ of gliomas (1). The World Health Organization (WHO) classification system separates oligodendrogliomas histopathologically into low-grade (WHO II, 77\%) and high-grade or anaplastic (WHO III, 23\%) tumor categories. Anaplastic oligoastrocytomas with necrosis are classified as glioblas-

Correspondence to: Professor Jian Yin, Department of Neurosurgery, The Second Affiliated Hospital of Dalian Medical University, 467 Zhongshan Road, Dalian, Liaoning 116044, P.R. China E-mail: dyeydr_yin@163.com

Key words: anaplastic oligodendrogliomas, meningioma, gliomas, arteriovenous malformations, mushroom-shaped growth, calcification, peritumoral edema tomas (WHO IV) (2). The median survival times of patients with WHO II and WHO III oligodendrogliomas are 9.8 and 3.9 years, respectively $(1,2)$, and 6.3 and 2.8 years, respectively, if mixed with astrocytes $(3,4)$. Surgical excision and postoperative adjuvant radiotherapy is the traditional therapy for oligodendroglioma; however, studies have observed that, among intracranial tumors, anaplastic oligodendrogliomas are particularly sensitive to chemotherapy, and the prognosis of patients treated with chemotherapy is more favorable than that of patients treated with radiotherapy (5-7). The preoperative differential diagnosis is, therefore, particularly important for therapeutic decisions and determining the prognosis of the patient. Magnetic resonance imaging (MRI) is widely discussed in the literature and employed in clinical practice (6); however, it may produce unclear results that can hinder a definitive diagnosis of oligodendroglioma. The present study describes a case of oligodendroglioma in which the results of the MRI examination were unclear, and pathological analysis was subsequently used to confirm a diagnosis of atypical anaplastic oligodendroglioma.

\section{Case report}

A 34-year-old man who suffered from headache and right upper-extremity weakness for 2 months was referred to The Second Affiliated Hospital of Dalian Medical University (Dalian, China) for medical care. Informed consent was obtained from the patient's family. The patient exhibited no impairment of consciousness, hearing, vision or sensory perception. Physical examinations revealed that the patient exhibited decreased hearing in his left ear, upper-extremity numbness and muscle strength decline. Axial computed tomography (CT) examination of the brain showed a large, high-density mass with calcification in the right parietal-temporal-occipital area, and the top of the mass pressed against the parieto-occipital subdural matter (Fig. 1A). An MRI scan revealed a mushroom-shaped mass, which was divided by the Sylvian fissure. The mass exhibited heterogeneous hypointensity under T1-weighted imaging (T1WI) and hyperintensity under T2WI and fluid-attenuated inversion 

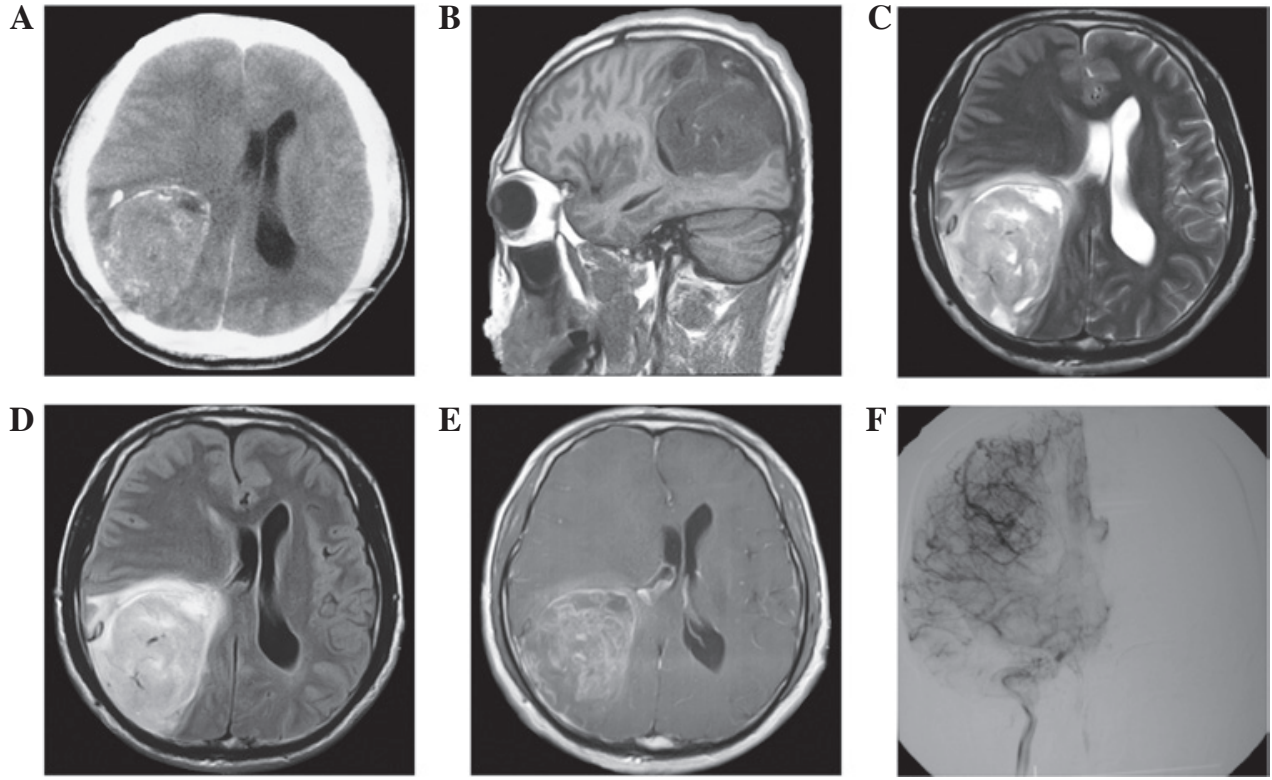

Figure 1. Imaging findings. (A) Axial computed tomography scan of the brain showed a large, high-density mass with calcification in the right parietal-temporal-occipital area; the top of the mass bulged onto the parieto-occipital subdural matter. (B) T1-weighted imaging (T1WI) and (C) T2WI magnetic resonance imaging scans revealed a mushroom-shaped growing mass, which was divided by the Sylvian fissure and appeared hypointense under T1WI and hyperintense under T2WI. (D) Axial T2 fluid-attenuated inversion recovery imaging revealed a hyperintense signal. (E) The mass was heterogeneously enhanced with prominent feeding arteries. (F) Frontal view of the internal carotid on digital subtraction angiography revealed no obvious mass lesion with large arteriovenous shunts or a vascular nidus mimicking a true arteriovenous malformation; however, the right middle cerebral artery and the draining vein were thickened.

A

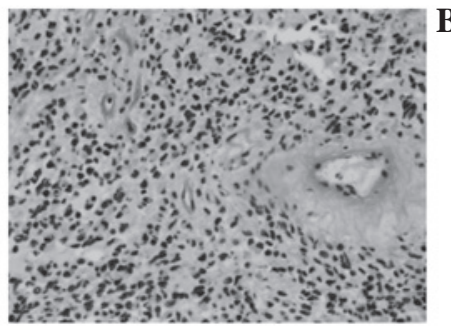

D

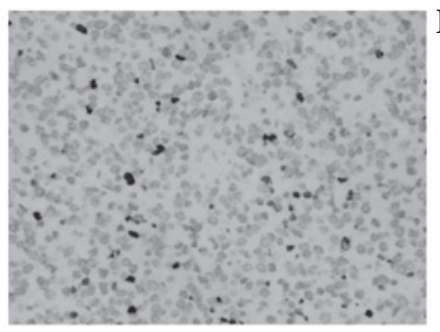

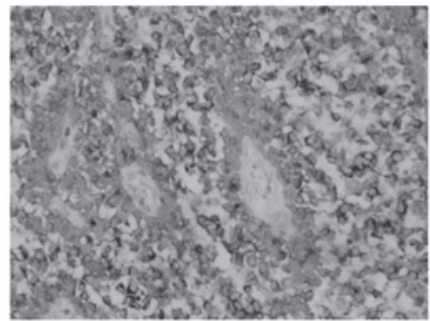

$\mathbf{E}$

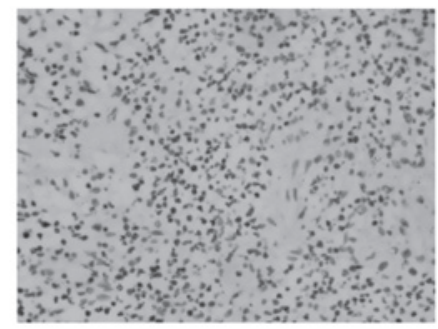

C
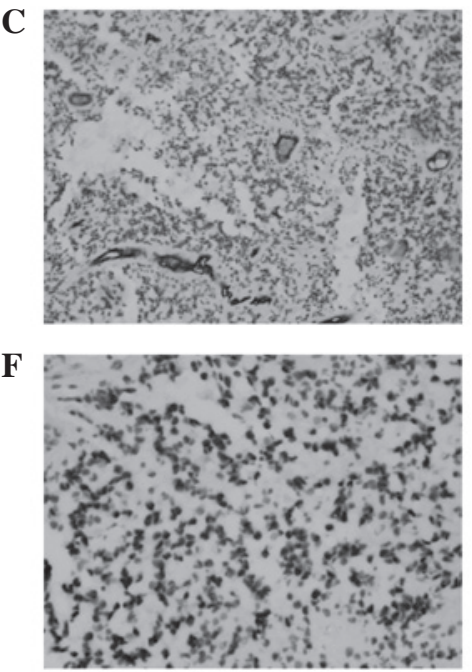

Figure 2. Pathological examination. (A) Hematoxylin and eosin-stained tissue (magnification, x100); (B) glial fibrillary acidic protein-positive tissue (magnification, x100); (C) cluster of differentiation 34-positive tissue, which indicates the proliferation of blood vessels (magnification, $\mathrm{x} 40$ ); (D) Ki-67, index 10\% (x100); (E) NeuN-negative tissue (magnification, x100); and (F) oligo-2-positive tissue (magnification, x100).

recovery imaging, which was caused by brain parenchyma deformation, with obvious peritumoral edema (Fig. 1B-D). The mass was heterogeneously enhanced following the intravenous administration of gadolinium, with prominent feeding arteries. In addition, the boundary of the tumor was enhanced, which appeared as blurriness under T2WI (Fig. 1E). The frontal view of the internal carotid artery, obtained using digital subtraction angiography (DSA), did not show an obvious mass with large arteriovenous shunts or a vascular nidus resembling a true arteriovenous malformation (AVM); however, the right middle cerebral artery and the draining vein were thickened. Based on the presurgical evaluation, it was suggested that the patient had a WHO grade II or III meningioma or AVM, as the radiological manifestations were unclear. Due to the patient's impaired function and the results of the radiological examination, a surgical resection was performed. During surgery, the partial dura mater was pushed outward by the tumor with high tension. Notably, soft, gray-red, cystic, highly liquid tissue with high-transmittance, with a jelly-like appearance, was expelled instead of adhering to the dura mater when cut radially. As expected, the insidious tumor growth boundaries were clear on the surrounding normal brain, which was covered by slightly yellowish particles on the membrane; however, the results of the pathological examination of the frozen section revealed 
an anaplastic glioma. The residual tumor, which was gelatinous and pinkish-gray in color, was subsequently separated in order to achieve subtotal excision. The immunostaining results (Fig. 2) were positive for glial fibrillary acidic protein (Fig. 2B); cluster of differentiation (CD) 34, which indicated the presence of blood vessel proliferation (Fig. 2C); and oligo-2, which is closely associated with oligodendrogliomas (Fig. 2F), but negative for NeuN (Fig. 2E). The Ki-67 index was $10 \%$, which indicated a high risk of recurrence (Fig. 2D). Accordingly, the postsurgical assessment confirmed that the mass was an anaplastic oligodendroglioma (WHO III), and the postoperative treatment proceeded with adjuvant radiation and chemotherapy. Radiographic follow-up evaluation after 6 months revealed no indications of tumor recurrence.

\section{Discussion}

The present case was notable due to the unclear radiological manifestations, which increased the difficulty of presurgical diagnosis. The rare growth pattern and morphology of the oligodendroglioma was also notable.

Oligodendrogliomas typically appear as hypodense (57-70\%) or isodense images under CT examination; however, the present case had a tendency towards a hyperdense appearance. It is established that intratumoral calcification is typical for meningioma; however, in cases of oligodendroglioma the incidence has been reported to be $50-90 \%(8,9)$. The shape of the calcification can be categorized as coarse, punctate or linear. The uneven edges of the calcified lesion manifest fairly discrete margins under CT imaging $(2,8)$. Thus, in the present case the calcification complicated the differential diagnosis. Additionally, the tumor was closely but non-aggressively associated with the calvaria, and the boundary was relatively clear. It was therefore not possible to differentiate oligodendroglioma from meningioma based upon CT alone.

Under MRI, the most prominent feature of the present tumor was the aforementioned mushroom-shape, which penetrated the cerebral cortex and arachnoid to the subdural space. The region near the posterior horn of the lateral ventricle represented the stalk of the mushroom. The cap of the mushroom-shaped tumor grew along the subdural space and connected with the endocranium to form a wide base resembling a meningioma under MRI. This type of growth pattern resembled two lesions at different sections. The stalk part exhibited invasive growth; conversely, the tumor capsule forming the cap of the mushroom, which was confirmed during surgery, was obvious.

There are numerous forms of tumor enhancement, including nodular and ring. Previous studies have suggested that a number of anaplastic oligodendrogliomas cannot be enhanced; however, a few low-grade (WHO II) tumors have been observed to be enhanced $(10,11)$. The absence of the dural tail sign and uneven enhancement under enhanced MRI suggest that the possibility of meningioma is limited.

Peritumoral edema is considered to be a key indicator of high-grade intracranial tumors; however, oligodendrogliomas are rarely associated with peritumoral vasogenic edema. Peritumoral edema cannot, therefore, be used as an indicator of tumor grading (12). In the present study, peritumoral edema was evident, but the pattern of edema was distinct from the finger-like pattern of edema associated with gliomas; therefore the evidence for a glioma was also insufficient.

Oligodendrogliomas are closely associated with AVMs, both in terms of histopathology and radiology (13). In a previous study, a patient was diagnosed with AVM and received embolization, yet developed a glioma 10 years later at the same site. Certain lesions appear to be oligodendrogliomas rich in vessels during preoperative diagnosis, but are subsequently pathologically confirmed as AVM through immunohistochemistry $(14,15)$. Vascular endothelial growth factor (VEGF) receptor, Ki-67 and CD34 have been associated with abnormal tumor angiogenesis, and the overexpression of VEGF, angiogenesis (contrast enhancement or endothelial hyperplasia) and absence of seizures are considered high-risk factors for poor prognosis (13). In the present study, the thick vascular enhancement on MRI was verified as a vein using DSA; this vein was before the normal venous drainage on the hemisphere surface from the sagittal view. Furthermore, the diameter was $\sim 2$-fold that of the normal venous drainage (Fig. 1D and F). Therefore, it may be useful to conduct DSA for cases in which MRI is unable to differentiate between oligodendroglioma and AVM.

In conclusion, the present study has described a type of mushroom-shaped anaplastic oligodendroglioma in the parietal-temporal-occipital region. This mushroom-shaped anaplastic oligodendroglioma, albeit extremely rare, is a potential source of misdiagnosis for meningioma and AVM.

\section{Acknowledgements}

The authors thank Mr. Hang Yin for his assistance in the collection of materials.

\section{References}

1. Engelhard HH, Stelea A and Mundt A: Oligodendroglioma and anaplastic oligodendroglioma: Clinical features, treatment, and prognosis. Surg Neurol 60: 443-456, 2003.

2. Louis DN, Ohgaki H, Wiestler OD, Cavenee WK, Burger PC Jouvet A, Scheithauer BW and Kleihues P: The 2007 WHO classification of tumours of the central nervous system. Acta Neuropathol 114: 97-109, 2007.

3. Hoang-Xuan K, Capelle L, Kujas M, Taillibert S, Duffau H, Lejeune J, Polivka M, Crinière E, Marie Y, Mokhtari K, et al: Temozolomide as initial treatment for adults with low-grade oligodendrogliomas or oligoastrocytomas and correlation with chromosome 1p deletions. J Clin Oncol 22: 3133-3138, 2004.

4. Shaw EG, Scheithauer BW, O'Fallon JR and Davis DH: Mixed oligoastrocytomas: A survival and prognostic factor analysis. Neurosurgery 34: 577-582, 1994.

5. Perry JR: Oligodendrogliomas: Clinical and genetic correlations. Curr Opin Neurol 14: 705-710, 2001.

6. Jenkinson MD, du Plessis DG, Smith TS, Joyce KA, Warnke PC and Walker C: Histological growth patterns and genotype in oligodendroglial tumours: Correlation with MRI features. Brain 129: 1884-1891, 2006.

7. Mason WP and Cairncross JG: Invited article: The expanding impact of molecular biology on the diagnosis and treatment of gliomas. Neurology 71: 365-373, 2008.

8. Lee YY and Van Tassel P: Intracranial oligodendrogliomas: Imaging findings in 35 untreated cases. AJR Am J Roentgenol 152: 361-369, 1989.

9. Dolinskas CA and Simeone FA: CT characteristics of intraventricular oligodendrogliomas. AJNR Am J Neuroradiol 8: 1077-1082, 1987 
10. Ginsberg LE, Fuller GN, Hashmi M, Leeds NE and Schomer DF: The significance of lack of MR contrast enhancement of supratentorial brain tumors in adults: Histopathological evaluation of a series. Surg Neurol 49: 436-440, 1998.

11. White ML, Zhang Y, Kirby P and Ryken TC: Can tumor contrast enhancement be used as a criterion for differentiating tumor grades of oligodendrogliomas? AJNR Am J Neuroradiol 26: 784-790, 2005 .

12. Spampinato MV, Smith JK, Kwock L, Ewend M, Grimme JD, Camacho DL and Castillo M: Cerebral blood volume measurements and proton MR spectroscopy in grading of oligodendroglial tumors. AJR Am J Roentgenol 188: 204-212, 2007.
13. Quon H, Hasbini A, Cougnard J, Djafari L, Lacroix C and Abdulkarim B: Assessment of tumor angiogenesis as a prognostic factor of survival in patients with oligodendroglioma. J Neurooncol 96: 277-285, 2010.

14. McKinney JS, Steineke T, Nochlin D and Brisman JL: De novo formation of large arteriovenous shunting and a vascular nidus mimicking an arteriovenous malformation within an anaplastic oligodendroglioma: Treatment with embolization and resection. J Neurosurg 109: 1098-1102, 2008.

15. Gmeiner M, Sonnberger M, Wurm G and Weis S: Glioblastoma with the appearance of arteriovenous malformation: Pitfalls in diagnosis. Clin Neurol Neurosurg 115: 501-506, 2013. 